\title{
Establishing a Nomogram for Stage IA-IIB Cervical Cancer Patients after Complete Resection
}

\author{
Hang Zhou ${ }^{1,2 \&}$, Xiong $\mathrm{Li}^{1 \&}$, Yuan Zhang ${ }^{3 \&}$, Yao Jia ${ }^{1}$, Ting Hu ${ }^{1}$, Ru Yang ${ }^{1}$, Ke-Cheng \\ Huang$^{1}$, Zhi-Lan Chen ${ }^{1}$, Shao-Shuai Wang ${ }^{1}$, Fang-Xu Tang ${ }^{1}$, Jin Zhou ${ }^{1}$, Yi-Le \\ $\mathrm{Chen}^{4}$, Li Wu ${ }^{4}$, Xiao-Bing Han ${ }^{5}$, Zhong-Qiu Lin ${ }^{6}$, Xiao-Mei Lu ${ }^{6}$, Hui Xing ${ }^{7}$, Peng- \\ Peng $\mathbf{Q u}^{8}$, Hong-Bing Cai ${ }^{9}$, Xiao-Jie Song ${ }^{10}$, Xiao-Yu Tian ${ }^{11}$, Qing-Hua Zhang ${ }^{12}$, \\ Jian Shen ${ }^{12}$, Dan Liu ${ }^{1}$, Ze-Hua Wang ${ }^{3}$, Hong-Bing Xu ${ }^{13}$, Chang-Yu Wang ${ }^{1}$, Ling \\ $\mathrm{Xi}^{1}$, Dong-Rui Deng ${ }^{1}$, Hui Wang ${ }^{1}$, Wei-Guo Lv ${ }^{14}$, Keng Shen ${ }^{15}$, Shi-Xuan Wang ${ }^{1}$, \\ Xing $\mathrm{Xie}^{14}$, Xiao-Dong Cheng ${ }^{14 *}$, Ding $\mathrm{Ma}^{1 *}$, Shuang $\mathrm{Li}^{1 *}$
}

\begin{abstract}
Background: This study aimed to establish a nomogram by combining clinicopathologic factors with overall survival of stage IA-IIB cervical cancer patients after complete resection with pelvic lymphadenectomy. Materials and Methods: This nomogram was based on a retrospective study on 1,563 stage IA-IIB cervical cancer patients who underwent complete resection and lymphadenectomy from 2002 to 2008 . The nomogram was constructed based on multivariate analysis using Cox proportional hazard regression. The accuracy and discriminative ability of the nomogram were measured by concordance index ( $C$-index) and calibration curve. Results: Multivariate analysis identified lymph node metastasis (LNM), lymph-vascular space invasion (LVSI), stromal invasion, parametrial invasion, tumor diameter and histology as independent prognostic factors associated with cervical cancer survival. These factors were selected for construction of the nomogram. The $C$-index of the nomogram was 0.71 (95\% CI, 0.65 to 0.77$)$, and calibration of the nomogram showed good agreement between the 5 -year predicted survival and the actual observation. Conclusions: We developed a nomogram predicting 5-year overall survival of surgically treated stage IA-IIB cervical cancer patients. More comprehensive information that is provided by this nomogram could provide further insight into personalized therapy selection.
\end{abstract}

Keywords: Cervical cancer - nomogram - clinicopathologic factor

Asian Pac J Cancer Prev, 16 (9), 3773-3777

\section{Introduction}

Cervical cancer is the third most common malignancy affecting women worldwide, with an estimated 500,000 new cases and more than 275,000 deaths in 2008 (Ferlay et al., 2010). In China, nearly 130,000 cervical cancer cases are newly diagnosed each year, and approximately 30,000 women die of cervical cancer every year (Nguyen et al., 2011).
Radical hysterectomy and pelvic lymph node dissection remain the primary therapeutic choices following the FIGO clinical staging system (Landoni et al., 1997; Waggoner, 2003). However, clinicopathologic factors other than FIGO stage, such as lymph node metastasis (LNM) and lymph-vascular space invasion (LVSI), have proven to be relevant to the prognosis of cervical cancer patients (Ho et al., 2004; Pecorelli et al., 2009; Singh et al., 2012). Although NCCN (Grochola et

Department of Obstetrics and Gynecology, ${ }^{1}$ Tongji Hospital, ${ }^{3}$ Union Hospital, Tongji Medical College, Huazhong University of Science and Technology, ${ }^{12}$ The Central Hospital of Wuhan, Wuhan, ${ }^{2}$ Nanjing Drum Tower Hospital, Nanjing University Medical School, Nanjing, ${ }^{5}$ The First Affiliated Hospital, Medical School of Xi'an Jiaotong University, Xi'an, ${ }^{7}$ Xiangyang Central Hospital, First Affiliated Hospital of Hubei University of Arts and Science. Xiangyang, Hubei, ${ }^{11}$ The First Affiliated Hospital of Henan University of Science and Technology, Luoyang, Henan, ${ }^{15}$ Department Obstetrics and Gynecology, Peking Union Medical College Hospital, Chinese Academy of Medical Sciences, Peking Union Medical College, Beijing, ${ }^{4}$ Department of Gynecologic Neoplasms, Hunan Province Tumor Hospital, The Affiliated Tumor Hospital of Central South University, Changsha, Department of Gynecologic Oncology, ${ }^{6}$ The Second Affiliated Hospital, Sun Yat-sen University, Guangzhou, Guangdong, ${ }^{9}$ Zhong Nan Hospital, Wuhan University, Wuhan, ${ }^{8}$ Tianjin Central Hospital for Gynecology and Obstetrics, Tianjin, ${ }^{10}$ Commercial Vocational Hospital, Wuhan, ${ }^{13}$ Shenzhen People's Hospital, the Second Clinical Hospital of Jinan University, Shenzhen, ${ }^{14}$ Women's Reproductive Health Laboratory of Zhejiang Province, Zhejiang, China ${ }^{\star} E q u a l$ contributors*For correspondence: dma@tjh.tjmu.edu.cn,lee5190008@126.com, chengxd001@163.com 
al., 2008)guidelines have suggested that adjuvant therapy should be applied to certain patients with intermediate or high risk factors after surgery, these factors remain controversial in many studies (Kamura et al., 1992; Yuan et al., 1998; Creasman and Kohler, 2004; Ho et al., 2004; Chang et al., 2009; Singh et al., 2012; Williams et al., 2015). Therefore, different opinions on the impact of prognostic factors between studies may lead to various selection criteria for adjuvant therapy, as suggested by researchers (Chang et al., 2009; Small et al., 2012).

Until now, most of the prognostic models for cervical cancer were based on the risk stratification method, which grouped together patients with similar prognoses (Kamura et al., 1992; Yuan et al., 1998; Ho et al., 2004; Singh et al., 2012). However, this method may ignore certain surgicalpathological factors. Nomograms are used to transform statistical equations to simplified graphs (Iasonos et al., 2008). In recent years, nomograms have been constructed for many malignancies, such as prostate cancer, bladder cancer and gastric cancer to obtain individualized prognostic information (Bochner et al., 2006; Walz et al., 2007; Han et al., 2012). Some of these nomograms have system (Sternberg, 2006; Wang et al., 2013). To our knowledge, a nomogram predicting overall survival for cervical cancer patients treated primarily with surgery has not been published thus far. Therefore, the aims of the study are to establish a nomogram for cervical cancer patients primarily treated with surgery.

\section{Materials and Methods}

\section{Patients}

Retrospective research was performed using the cervical cancer database, which stores the information of 10,897 patients (http://clinicaltrials.gov). This study was approved by the Ethics Committee of Tongji Hospital, Tongji Medical College, Huazhong University of Science and Technology, P. R. China.

Inclusion criteria for patients in this study were as follows: stage IA-IIB cervical cancer patients treated with radical surgery, no history of other malignancies, no preoperative adjuvant therapy, no residual macroscopic or microscopic tumor and no missing values. The exclusion criterion for patients in this study was: rare histological type of cervical cancer including small cell carcinoma. We extracted patient demographics and clinicopathologic characteristics (FIGO stage, number of positive lymph node, LVSI, depth of stromal invasion, parametrial invasion, tumor diameter, grade and histology). Tumor diameter was determined by measuring the longest diameter using magnetic resonance imaging (MRI), computed tomography (CT), or ultrasound. Depth of stromal invasion was categorized as inner $1 / 3$, middle $1 / 3$ or outer $1 / 3$ following the postoperative pathological reports. Tumor grade classification was guided by Border system and was listed as well, moderate or poor differentiation. Histology was simply classified as either squamous or non-squamous cell carcinoma. The number of positive lymph nodes was categorized into 3 groups: 0,1 and $\geq 2$. Diagnosis was confirmed by two experienced shown to be more reliable than the traditional staging

pathologists.

The administration of adjuvant therapy was determined by attending physicians. Generally, patients with LNM or parametrial invasion were considered for administration of adjuvant concurrent chemoradiation, and patients with deep stromal invasion, bulky disease $(>4 \mathrm{~cm})$ or LVSI were considered for adjuvant chemotherapy or radiotherapy.

Patients were suggested to have postoperative evaluation every 3 months in the first year, every 6 months during the next four years and once a year thereafter. OS time was calculated from the time of surgery to the date of death or last contact. Follow-up data were collected from hospital records or by phone calls for patients who were lost to regular follow-up.

\section{Statistical analysis}

Data were first analyzed by standard description statistics. Survival curves were estimated by the KaplanMeier method and compared using the log-rank test. Multivariate analysis was performed by Cox regression analysis.

The nomogram was constructed based on the results of the Cox regression. Final variable selection was performed by a backward stepwise process following Akaike information criterion (AIC), and the model with the lowest AIC was selected (Harrell et al., 1996). Age was modeled with restricted cubic splines to accommodate a nonlinear relationship with outcome. The nomogram was internally validated by discrimination and calibration. Discrimination was assessed by concordance

Table 1. Clinicopathologic Characteristics of Patients Enrolled

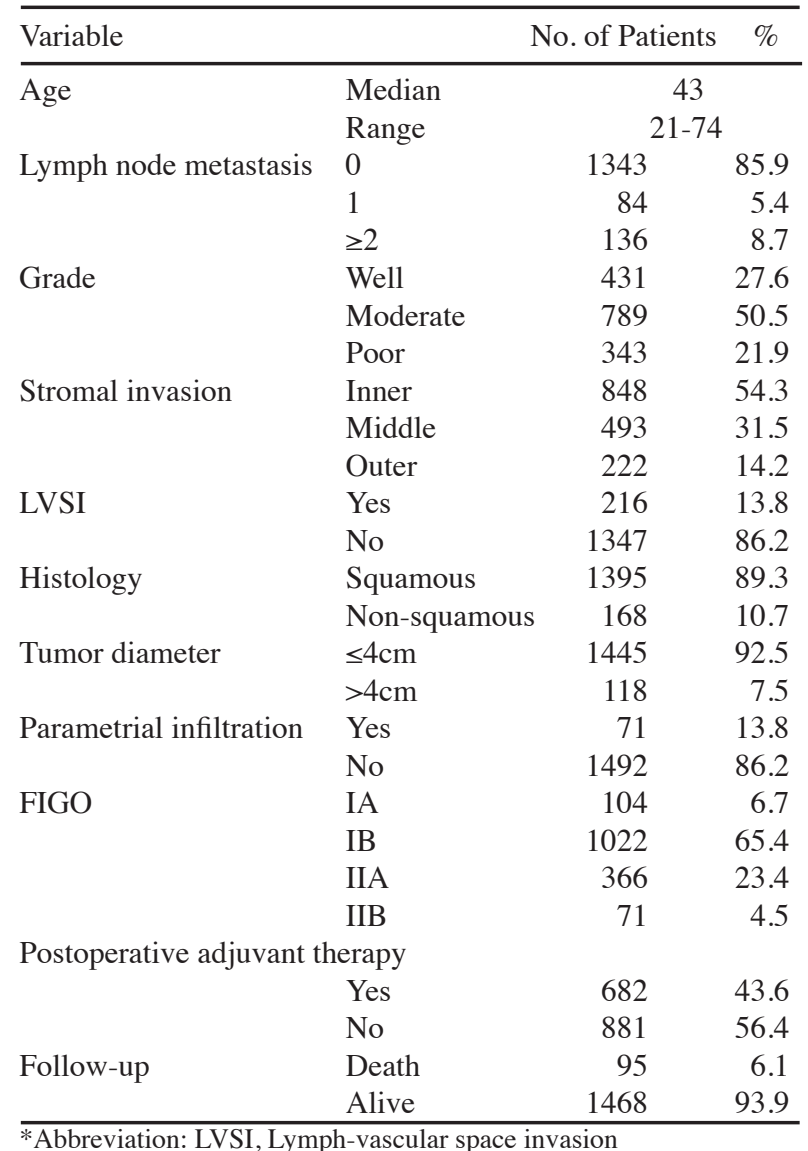

3774 Asian Pacific Journal of Cancer Prevention, Vol 16, 2015 
Table 2. COX Proportional Hazard Model for Cervical Cancer Patients

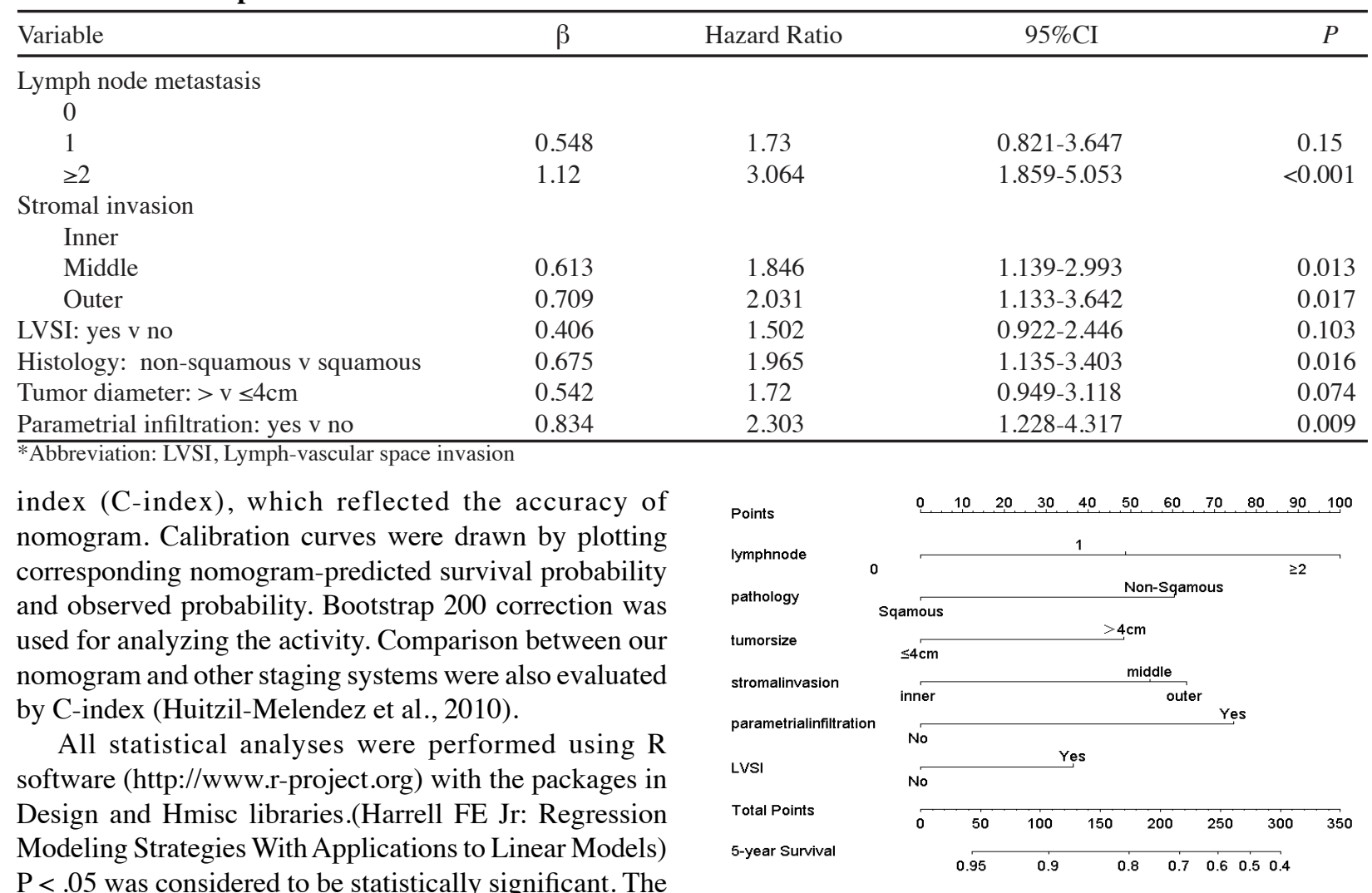
$P<.05$ was considered to be statistically significant. The related computerized programming language was listed in a previous study published by Wang et al. (2013) and was reprogrammed for the present study.

\section{Results}

\section{Clinicopathologic characteristics of patients}

A total of 1,563 cervical cancer patients were included in this study. Most patients had stage IB disease $(65.4 \%$, $\mathrm{n}=1,022)$ and squamous cell carcinoma $(89.3 \%, \mathrm{n}=1,395)$, and 682 of these patients received postoperative therapy $(43.6 \%)$. Patients without LNM predominated and accounted for 1,343 patients $(85.9 \%)$. Patients with bulky disease $(>4 \mathrm{~cm})$ were reported in only 118 patients (7.5\%). Detailed descriptions of patients' characteristics are shown in Table 1.

Selection of prognostic factors for construction of the nomogram

The median follow-up time was 42 months (range, 1-110 months), and the median time to death was 25 months (range, 6-83 months). The 5-year OS rate was $92.8 \%$. The results of univariate analysis are shown in Appendix Table S1. Multivariate analysis revealed that LNM, LVSI, stromal invasion, parametrial invasion, tumor diameter and histology were independent prognostic factors with the lowest AIC of 1,271 and is listed in Table 2 .

\section{Nomogram for $O S$}

The prognostic nomogram using all significant independent prognostic factors for OS is shown in Figure 1. The C-index for the nomogram is 0.71 (95\% CI, 0.65 to 0.77$)$. The calibration curve reveals good agreement

Figure 1. Nomogram for Cervical Cancer Patients Treated with Primary Surgery. To calculate the probability of 5 year overall survival, the value of each clinicopathologic factors should be determined by drawing a vertical line from the factor to the points. Sun the points of each factor and draw a straight line from total points to the 5 year survival line to obtain 5 year survival estimates

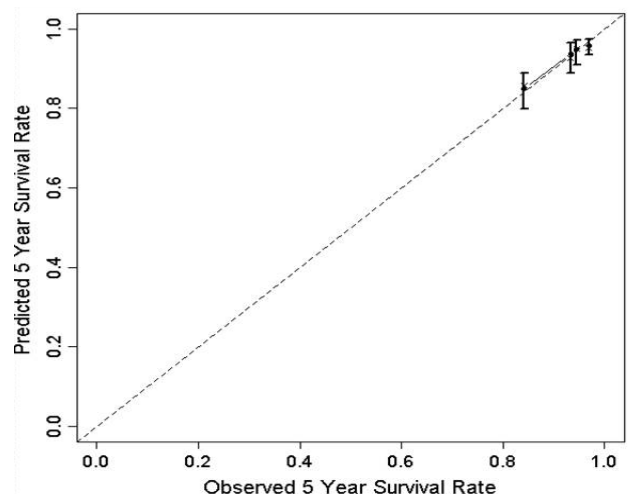

Figure 2. Calibration Curve for the 5 Year Overall Survival from Nomogram. The dashed line represents the ideal fit. The nomogram predicted probability of 5 year overall survival is plotted on $\mathrm{x}$-axis, and the actual 5 year overall survival is plotted on $y$-axis

between prediction of the nomogram and observation, as shown in Figure 2.

\section{Discussion}

The FIGO staging system has been commonly used for clinical evaluation of cervical cancer patients.(Pecorelli et al., 2009) However, its utility is low in guiding adjuvant therapy (Hricak et al., 2005). Although clinicopathologic 
factors have been suggested to guide postoperative adjuvant therapy selection, controversy surrounding the independence of these factors for prognosis of cervical cancer patients still exists (Chang et al., 2009; Small et al., 2012). These different results may be due to the heterogeneity of the population enrolled in different studies (Kamura et al., 1992; Yuan et al., 1998; Ho et al., 2004; Singh et al., 2012). For example, the Yuan model enrolled stage IB-IIA squamous cell carcinoma patients, while other models chose patients of different stages and histology.

Studies have shown that nomograms may be more accurate than conventional staging systems for prognostic prediction in many cancers, such as cholangiocarcinoma and bladder cancer (Sternberg, 2006; Wang et al., 2013). Nomograms that have been constructed to focus on cervical cancer patients of advanced stages who are receiving concurrent chemoradiotherapy (Tseng et al., 2010; Kang et al., 2012). However, no nomogram emphasizing on patients primarily treated with surgery has been developed thus far. This nomogram reached a C-index of 0.71 and performed well in the calibration curve.

However, this study has several limitations. First, this research was retrospective, although multiple excellent gynecologic centers in China participated in the study. Mistakes or bias in data collection and inputting phase may exist, although related training and double-check were applied throughout the study. The participation of different centers enabled the enlargement of the study population and may reveal small differences of certain prognostic factors. However, criteria and regiments of postoperative treatment among centers differed, which may affect the variable's magnitude of effect. Second, whether patients with preoperative adjuvant therapy should be excluded warrants further discussion. Our previous studies showed that preoperative adjuvant therapy may change the distribution of clinicopathologic factors, especially in intermediate factors (Hu et al., 2012; Hu et al., 2013). Third, the nomogram was only internally validated, which may produce the theoretical probability of over-estimation. Therefore, data from other centers need to be collected for external validation.

In summary, this is the first nomogram constructed for cervical cancer patients treated primarily with surgery. The nomogram was developed based mainly on stage IB patients and internally validated by bootstrap. The application of the nomogram will be more widespread when its limitations are addressed in the future.

\section{Acknowledgements}

This study was endorsed by the Key Basic Research and Development Program Foundation of China (973 Program; No. 2009CB521808) and was supported by grants from the National Natural Science Foundation of China (NO. 81230038; 81230052; 30973472; 81001151; 81071663; 30973205; 30973184; 81172464; 81101964), National Major Science and Technology Project (No. 2009ZX09103-739) and Fundamental Research Funds for the Central Universities (No. 20620140715).

\section{References}

Bochner BH, Kattan MW, Vora KC (2006). Postoperative nomogram predicting risk of recurrence after radical cystectomy for bladder cancer. J Clin Oncol, 24, 3967-72.

Chang SJ, Kim WY, Yoo SC, et al (2009). A validation study of new risk grouping criteria for postoperative treatment in stage IB cervical cancers without high-risk factors: rethinking the gynecologic oncology group criteria. Eur $J$ Obstet Gynecol Reprod Biol, 147, 91-6.

Creasman WT, Kohler MF (2004). Is lymph vascular space involvement an independent prognostic factor in early cervical cancer? Gynecol Oncol, 92, 525-9.

Ferlay J, Shin HR, Bray F, et al (2010). Estimates of worldwide burden of cancer in 2008: GLOBOCAN 2008. Int J Cancer, 127, 2893-917.

Grochola LF, Greither T, Taubert H, et al (2008). The stem cell-associated Hiwi gene in human adenocarcinoma of the pancreas: expression and risk of tumour-related death. $\mathrm{Br} J$ Cancer, 99, 1083-8.

Han DS, Suh YS, Kong SH, et al (2012). Nomogram predicting long-term survival after $\mathrm{d} 2$ gastrectomy for gastric cancer. J Clin Oncol, 30, 3834-40.

Harrell FE, Jr., Lee KL, Mark DB (1996). Multivariable prognostic models: issues in developing models, evaluating assumptions and adequacy, and measuring and reducing errors. Stat Med, 15, 361-87.

Harrell FE Jr (2001). Regression modeling strategies with applications to linear models LR, and survival analysis, volume 19. New York, NY, Springer-Verlag.

Ho CM, Chien TY, Huang SH, et al (2004). Multivariate analysis of the prognostic factors and outcomes in early cervical cancer patients undergoing radical hysterectomy. Gynecol Oncol, 93, 458-64.

Hricak H, Gatsonis C, Chi DS, et al (2005). Role of imaging in pretreatment evaluation of early invasive cervical cancer: results of the intergroup study American college of radiology imaging network 6651-gynecologic oncology group 183. J Clin Oncol, 23, 9329-37.

$\mathrm{Hu}$ T, Li S, Chen Y, et al (2012). Matched-case comparison of neoadjuvant chemotherapy in patients with FIGO stage IB1-IIB cervical cancer to establish selection criteria. Eur $J$ Cancer, 48, 2353-60.

Hu T, Wu L, Xing H, et al (2013). Development of criteria for ovarian preservation in cervical cancer patients treated with radical surgery with or without neoadjuvant chemotherapy: a multicenter retrospective study and meta-analysis. Ann Surg Oncol, 20, 881-90.

Huitzil-Melendez FD, Capanu M, O'Reilly EM, et al (2010). Advanced hepatocellular carcinoma: which staging systems best predict prognosis? J Clin Oncol, 28, 2889-95.

Iasonos A, Schrag D, Raj GV, et al (2008). How to build and interpret a nomogram for cancer prognosis. J Clin Oncol, 26, 1364-70.

Kamura T, Tsukamoto N, Tsuruchi N, et al (1992). Multivariate analysis of the histopathologic prognostic factors of cervical cancer in patients undergoing radical hysterectomy. Cancer, 69, 181-6.

Kang S, Nam BH, Park JY, et al (2012). Risk assessment tool for distant recurrence after platinum-based concurrent chemoradiation in patients with locally advanced cervical cancer: a Korean gynecologic oncology group study. J Clin Oncol, 30, 2369-74.

Landoni F, Maneo A, Colombo A, et al (1997). Randomised study of radical surgery versus radiotherapy for stage Ib-IIa cervical cancer. Lancet, 350, 535-40.

Nguyen TL, Nguyen DC, Nguyen TH, et al (2011). Survey- 

based cancer mortality in the Lao PDR, 2007-08. Asian Pac J Cancer Prev, 12, 2495-8.

Pecorelli S, Zigliani L, Odicino F (2009). Revised FIGO staging for carcinoma of the cervix. Int J Gynaecol Obstet, 105, 107-8.

Singh P, Tripcony L, Nicklin J (2012). Analysis of prognostic variables, development of predictive models, and stratification of risk groups in surgically treated FIGO early-stage (IAIIA) carcinoma cervix. Int J Gynecol Cancer, 22, 115-22.

Small W, Jr., Strauss JB, Jhingran A, et al (2012). ACR appropriateness criteria( $\mathrm{R})$ definitive therapy for early-stage cervical cancer. Am J Clin Oncol, 35, 399-405.

Sternberg CN (2006). Are nomograms better than currently available stage groupings for bladder cancer? J Clin Oncol, 24, 3819-20.

Tseng JY, Yen MS, Twu NF, et al (2010). Prognostic nomogram for overall survival in stage IIB-IVA cervical cancer patients treated with concurrent chemoradiotherapy. Am J Obstet Gynecol, 202, 174 e1-7.

Waggoner SE (2003). Cervical cancer. Lancet, 361, 2217-25.

Walz J, Gallina A, Saad F, et al (2007). A nomogram predicting 10-year life expectancy in candidates for radical prostatectomy or radiotherapy for prostate cancer. J Clin Oncol, 25, 3576-81.

Wang Y, Li J, Xia Y, et al (2013). Prognostic nomogram for intrahepatic cholangiocarcinoma after partial hepatectomy. J Clin Oncol, 31, 1188-95.

Williams NL, Werner TL, Jarboe EA, et al (2015). Adenocarcinoma of the cervix: should we treat it differently? Curr Oncol Rep, 17, 440.

Yuan CC, Wang PH, Lai CR, et al (1998). Prognosis-predicting system based on factors related to survival of cervical carcinoma. Int J Gynaecol Obstet, 63, 163-7. 